\title{
OBSERVATIONS OF DISCRETE SOURCES AT 3-CM WAVELENGTH USING A MASER*
}

\author{
L. E. ALSOP AND J. A. GIORDMAINE† \\ Columbia University, New York, U.S.A. \\ C. H. MAYER \\ U.S. Naval Research Laboratory, Washington, D.C., U.S.A. \\ C. H. TOWNES \\ Columbia University, New York, U.S.A.
}

\section{INTRODUCTION}

The new types of low-noise radio-frequency amplifiers now under development hold great promise for improving and extending radio astronomical observations. A solid-state maser, designed and built at Columbia University, has been used with the U.S. Naval Research Laboratory's 50-foot reflector since 1958 April. The maser is the 3-level type [1], with ruby [2] as the paramagnetic medium. Initially, an rms radiometer output fluctuation of about $0.1^{\circ} \mathrm{K}$ was realized with a $5^{\mathrm{s}}$ output time constant. Subsequent improvements of the maser and of the associated circuitry have made it possible to observe with an rms output fluctuation of $0.04^{\circ} \mathrm{K}$ for a $5^{\text {s }}$ output time constant. These figures represent improvement factors of about 5 and 13 respectively over the radiometer without the maser. These significant improvements in sensitivity have made possible continuum observations which could not previously have been made with the NRL apparatus except by unwieldy averaging techniques.

\section{OBSERVATIONS}

The observations reported have all been made with a $5^{\text {s }}$ output time constant. The method of calibrating the antenna temperature has been described in [3] along with the previous measurement of antenna gain, which was relied on to convert the measured antenna temperatures to apparent black-body temperatures and to flux densities. The antenna-temperature

* Work at Columbia University was supported by the U.S. Army Signal Corps, the U.S. Air Force Office of Scientific Research, Air Research and Development Command, and the U.S. Navy Office of Naval Research. Part of this material was presented to the American Astronomical Society in June 1958 and an abstract will appear in the Astronomical Journal.

† Esso Research Education Foundation Fellow, 1957-58. 
measurements of the more intense radio sources made at the time of the antenna-gain measurement and at the present time agree to within a few per cent. Because of the rapid improvement in system performance over the three-month interval, the observations have been made over a range of sensitivities, which is apparent from the illustrations. The wavelength has also been changed several times, either to conform to the observing program or to avoid radar interference.

The radio source Cygnus A (I.A.U. 19N4A) was observed to provide an antenna diagram and an antenna-pointing reference. In Fig. 1, a drift scan
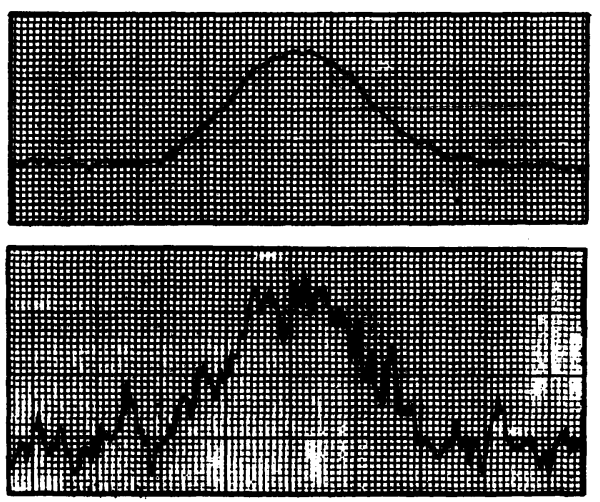

Fig. 1. Cygnus A drift curves with the 50foot reflector and a 5-second output time constant. Upper: maser preamplifier at $3.2-\mathrm{cm}$ wavelength, 1958 July. Lower: without maser at $3.15-\mathrm{cm}$ wavelength, 1956 September. using the maser at $3.2-\mathrm{cm}$ wavelength (antenna temperature $4.6^{\circ} \mathrm{K}$ ) is compared with a drift scan made in 1956 at $3.15 \cdot \mathrm{cm}$ wavelength (antenna temperature $4.7^{\circ} \mathrm{K}$ ) [4] with the same antenna and radiometer but without the maser. The point source flux density corresponding to the $4.6^{\circ} \mathrm{K}$ antenna temperature is $1.24 \times 10^{-24}$ watts $\mathrm{m}^{-2}(\mathrm{c} / \mathrm{s})^{-1}$. The probable error in the present estimate of flux density is about 9 per cent. The drift scans through Cygnus A indicate a halfintensity width for the antenna diagram of 0.15 degrees. This source has been previously observed by Haddock and McCullough at $3.15-\mathrm{cm}$ wavelength [5] and by Razin and Plechkov [6] at $3.2-\mathrm{cm}$ wavelength.

Venus was observed on 1958 April $18-19$ at $3.37-\mathrm{cm}$ wavelength. The measured antenna temperature was $0.45^{\circ} \mathrm{K}$, which corresponds to an apparent black-body temperature based on the diameter of the visible disk of $575{ }^{\circ} \mathrm{K}$ with a probable error of 10 per cent. These observations made 80 days after conjunction are not significantly different from the observations made with the same reflector near the conjunction of 1956 at $3.15-\mathrm{cm}$ wavelength [3].

A number of single observations of Jup.ter at $3.18-\mathrm{cm}$ wavelength (Fig. 2) gave an antenna temperature of $0.51^{\circ} \mathrm{K}$ corresponding to an apparent blackbody temperature based on the mean diameter of the visible disk of $165^{\circ} \mathrm{K}$

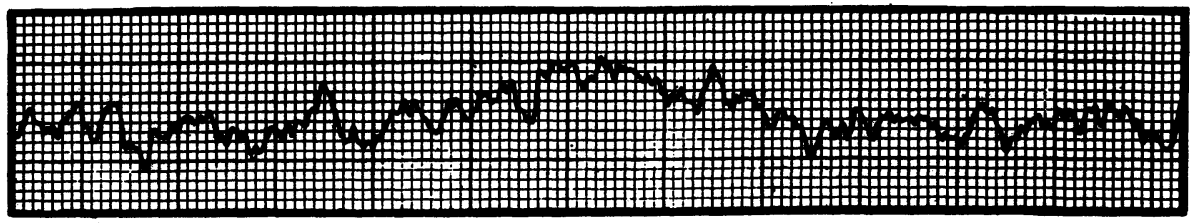

Fig. 2. Jupiter drift curve, 1958 April. 
with a probable error of about 10 per cent. The previous observations of Jupiter at $3.15-\mathrm{cm}$ wavelength [7], which used the same antenna and calibration techniques but required the point-by-point averaging of $45 \mathrm{drift}$ scans to obtain a single measurement with a probable error of 12 per cent, gave an apparent black-body temperature of $145^{\circ} \mathrm{K}$. An inspection of the present single observations indicates that any variation in apparent black-body temperature with rotation of Jupiter is less than 10 per cent.

The radio source Virgo A (NGC 4486, I.A.U. 12N1A) has now been observed (Fig. 3). At $3.37-\mathrm{cm}$ wavelength the maximum antenna temperature was

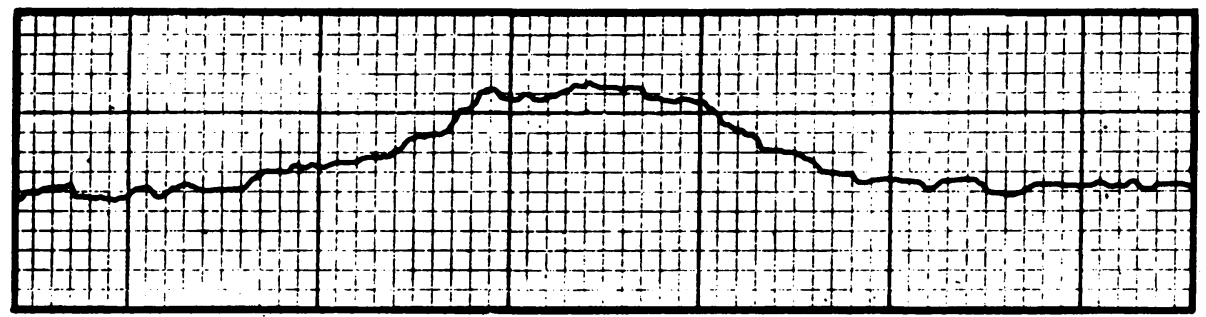

FIG. 3. Virgo A drift curve, 1958 July.

$1.25^{\circ} \mathrm{K}$, which would correspond to a point source flux density of $3.4 \times 10^{-25}$ watts $\mathrm{m}^{-2}(\mathrm{c} / \mathrm{s})^{-1}$. The angular extent of the Virgo A source has been measured at 3-m wavelength [8] as $2 \frac{1}{2} \times 5$ minutes of arc, which is appreciable compared to the beamwidth of the 50 -foot reflector at $3.37-\mathrm{cm}$ wavelength. Assuming that the $3-\mathrm{cm}$ source is the same size as the long wavelength source, the point source flux can be corrected upward following Seeger, Westerhout, and van de Hulst [9]. The resulting flux density for a uniformly bright source is $3.6 \times 10^{-25}$ watts $\mathrm{m}^{-2}(\mathrm{c} / \mathrm{s})^{-1}$ and for a source with Gaussian brightness distribution $3.9 \times 10^{-25}$ watts $\mathrm{m}^{-2}(\mathrm{c} / \mathrm{s})^{-1}$. The probable error on the determination of flux density is about 10 per cent not including the uncertainty in the angular extent of the source.

\section{THE MASER}

A three-level maser* amplifier with ruby [2] as the paramagnetic amplifying medium was used in the observations. The mechanism of the three-level maser, described elsewhere [1], will be outlined here only briefly.

In Fig. 4 the energy-level scheme of the lowest-lying levels of $\mathrm{the}^{\mathrm{Cr}} \mathrm{r}^{+3}$ ion in ruby $\left(\mathrm{Al}_{2} \mathrm{O}_{3} \times x \mathrm{Cr}_{2} \mathrm{O}_{3}\right)$ are illustrated. At thermal equilibrium, in the absence of radiation, the population of level 3 is related to that of level 2 by the Boltzmann ratio, and

$$
n_{2}-n_{3} \approx \frac{N h \nu_{23}}{3 k T}
$$

where $N$ is the total population of levels 1,2 , and 3 . If a sufficient amount

* Microwave amplification by stimulated emission of radiation. 
of radiation at the frequency $\nu_{13}$ is coupled to the system, the populations of levels 1 and 3 can be made equal. For this situation, the popuiation difference is given by

$$
n_{2}-n_{3} \sim \frac{N h}{3 k T}\left(\frac{\nu_{23}-\nu_{12}}{2}\right) .
$$

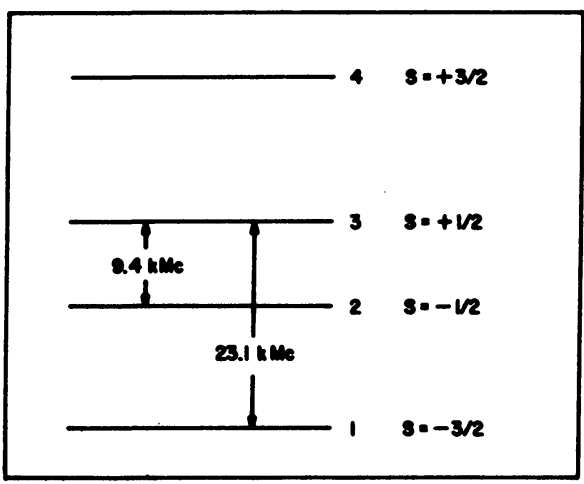

Fig. 4. Energy level diagram of $\mathrm{Cr}^{+3}$ in ruby for $H=3900$ oersteds, $\phi=58$ degrees.

For simplicity it is assumed here that the relaxation times for the transitions 2 to 3 and 1 to 2 , which determine the rate of return of a disturbed system to thermal equilibrium, are the same. Therefore, if $\nu_{12}>\nu_{23}$ the population difference $n_{2}-n_{3}$ becomes negative, and radiation at the frequency $\nu_{23}$ coupled to the system is amplified by stimulated emission.

The circuit analogue of a maser amplifier is a negative-resistance amplifier, and in common with this type of amplifier the (voltage gain)bandwidth product is approximately a constant. The gain and bandwidth are related exactly by (2):

$$
\begin{aligned}
& (\sqrt{G}-1) B=2 \nu_{23}\left(\frac{1}{\left|Q_{M}\right|}-\frac{1}{Q_{0}}\right) \\
& (\sqrt{G}+1) B=\frac{2 \nu_{23}}{Q_{E}},
\end{aligned}
$$

where $G$ is the amplifier power gain, $B$ is the amplification bandwidth, $Q_{0}$ is the quality factor of the uncoupled cavity, $Q_{E}$ is the quality factor associated with the external coupling of the cavity, and $Q_{\boldsymbol{M}}$ is the quality factor associated with the amplifying material, and is negative.

In terms of the population difference $n_{3}-n_{3}(1)$,

$$
Q_{x} \sim \frac{h V \Delta \nu}{32 \pi \beta^{2}\left(n_{2}-n_{3}\right)},
$$

where $V$ is the cavity volume, with the paramagnetic medium completely filling the cavity, $\Delta \nu$ is the width at half-intensity of the absorption line at $\nu_{23}$, and $\beta$ is the Bohr magneton $\left(0.93 \times 10^{-20} \mathrm{ergs} /\right.$ gauss $)$.

From (1), (2), and (3), where $\left|Q_{x}\right|<Q_{0}$ it is seen that the gain-bandwidth product varies inversely with temperature. For this reason, and to allow saturation of levels 1 and 3 with the minimum amount of pumping power, the maser was operated at $1.4^{\circ} \mathrm{K}$.

The amplifier gain can be varied by adjusting the cavity coupling, and the gain and the bandwidth are chosen to provide the best over-all system noise figure consistent with (2). 
The increased sensitivity of the radiometer with the maser preamplifier arises from the low-noise power introduced by the maser itself. For the realizable situation in which the population of the upper state of the amplifying transition is much greater than that of the lower state, the equivalent noise temperature at the input to the maser is expected [10] to approach $h \nu_{23} / k$ or $0.4^{\circ} \mathrm{K}$ at $\nu_{23}=9400 \mathrm{Mc} / \mathrm{s}$, a limit imposed by spontaneous emission processes. Experimental measurements of maser noise levels [11] tend to support this prediction.

In the present apparatus, the equivalent noise temperature at the input to the maser is estimated to be about $80^{\circ} \mathrm{K}$. This high temperature is introduced by transmission-line losses and antenna spillover.

\section{AMPLIFIER APPARATUS AND PERFORMANCE}

The ruby crystal was mounted in a microwave cavity resonant to both the signal and pumping frequencies. Observations were made at frequencies in the range from 8900 to $9500 \mathrm{Mc} / \mathrm{s}$. The crystal, cavity, and supporting waveguide were immersed in liquid helium, which was pumped down to provide a temperature of $1.4^{\circ} \mathrm{K}$. The stainless-steel dewar had a capacity of about 3 liters, allowing a 15-hour observation period on a single charge of helium.

Two cavities and two crystals were used. The first, in which the ruby filled about 12 per cent of the cavity volume, had a gain-bandwidth product of about $10 \mathrm{Mc} / \mathrm{s}$. With this arrangement, an rms noise-fluctuation level of $0.07^{\circ} \mathrm{K}$ was obtained for a 5 -second averaging time. Most of the observations were made at a fluctuation level of $0.1^{\circ} \mathrm{K}$. The second cavity, filled almost completely with ruby, provided a gain-bandwidth product of about $100 \mathrm{Mc} / \mathrm{s}$ in the laboratory under optimum conditions. Under actual operating conditions, in which a permanent magnet with smaller pole faces than those of the large electromagnet of the laboratory was used to conserve weight, the increased inhomogeneity of the applied field reduced the gain-bandwidth product to about $50 \mathrm{Mc} / \mathrm{s}$. In this case the rms noise fluctuation was $0.04{ }^{\circ} \mathrm{K}$ for a 5 -second averaging time. In each case, the bandwidth of the i-f amplifier following the maser was $5.5 \mathrm{Mc} / \mathrm{s}$.

The ruby was used in a magnetic field of about 3900 oersteds provided by a permanent magnet and auxiliary coils. The angle between the crystal symmetry axis and the magnetic field, which determines the spacing of the energy levels [12, 13], was about 58 degrees.

Since the maser used a reflection-type cavity, a ferrite circulator was used to isolate the maser from the high-temperature radiation from the mixer crystal. The modified Dicke radiometer in which the maser was used as an $r-f$ amplifier has been described elsewhere [3]. The maser, the local oscillator, and the i-f amplifier were mounted near the focus of the NRL 50-foot reflector to minimize transmission-line losses.

This first application of a maser amplifier demonstrates the substantial improvement in sensitivity afforded by the maser and the feasibility of using the maser as a tool in radio astronomy. 


\section{REFERENCES}

[1] Bloembergen, N. Phys. Rev. 104, 324, 1956.

[2] Makhov, G., Kikuchi, C., Lambe, J., and Terhune, R. W. Phys. Rev. 109, 1399, 1958.

[3] Mayer, C. H., McCullough, T. P., and Sloanaker, R. M. Ap. J. 127, 1, 1958.

[4] McCullough, T. P., and Sloanaker, R. M. Private communication.

[5] Haddock, F. T., and McCullough, T. P. A.J. 60, 161, 1955.

[6] Razin, V. A., and Plechkov, V. M. Radio Astronomy (I.A.U. Symposium No. 4, 1955). Cambridge, England, 1957, p. 155.

[7] Mayer, C. H., McCullough, T. P., and Slcanaker, R. M. Ap. J. 127, 11, 1958.

[8] Mills, B. Y. Aust. J. Phys. 6, 452, 1953.

[9] Seeger, C. L., Westerhout, G., and van de Hulst, H. C. B.A.N. 13, 89, 1956.

[10] Shimoda, K., Takahashi, H., and Townes, C. H. J. Phys. Soc. Japan, 12, 686, 1957.

[11] McWhorter, A. L., and Arams, F. R. Proc. I.R.E. 46, 913, 1958.

[12] Geusic, R. Phy8. Rev. 102, 1252, 1956.

[13] Schulz-Dubois, E. O., Degrasse, R. W., and Scovil, H. E. D. Symposium on Solid-State Masers (U.S. Army Signal Research and Development Laboratory, Fort Monmouth, N.J.), June 1958. 